\title{
On the influence of the air flow resistivity on the measurement of the dynamic stiffness of underlays for floating floors
}

\author{
Martin Schmelzer, Volker Wittstock*, Heinrich Bietz, and Spyros Brezas \\ Physikalisch-Technische Bundesanstalt (PTB), Bundesallee 100, 38116 Braunschweig, Germany
}

Received 2 December 2020, Accepted 27 January 2021

\begin{abstract}
The dynamic stiffness of underlays is a required quantity to predict the reduction of impact and airborne noise transmitted through floating floors. The measurement of the dynamic stiffness is standardized in ISO 9052-1 using a floating floor section of $200 \mathrm{~mm}$ edge length. If open pore insulating materials are used for the underlay, the air flow and its flow resistivity have a significant impact on the dynamic stiffness. The standard ISO 9052-1 therefore calculates the dynamic stiffness based on three distinct intervals of the flow resistivity. This contribution analyses and discusses the mathematical origin and practical consequences of these three standardized intervals and proposes an improvement for a revision of the standard. The current physical model is expanded to examine if the results, that are gained from the geometrically reduced systems of the standard, can be transferred to room sized systems.
\end{abstract}

Keywords: Underlays for floating floors, Airflow resistivity, Dynamic stiffness measurement

\section{Introduction}

Floating floor systems are widely used in buildings to reduce the transmission of impact and airborne noise. The underlay acts as a spring which decouples the vibration of the floating floor from the bare floor. This decoupling occurs at frequencies significantly higher than the resonance frequency $f_{\mathrm{r}}$ of the system, which can easily be calculated from the mass per unit area of the floating floor $m^{\prime}$ and the dynamic stiffness per unit area of the underlay $s$, based on the model of a simple oscillator:

$$
f_{\mathrm{r}}=\frac{1}{2 \pi} \sqrt{\frac{s^{\prime}}{m^{\prime}}} .
$$

To achieve a large frequency range of vibration decoupling, this resonance frequency $f_{\mathrm{r}}$, and thereby the dynamic stiffness $s^{\prime}$, must be as low as possible. Often, open pore insulating materials are used to realize this.

That makes the dynamic stiffness itself and its measurement very important for the prediction of the acoustic properties of buildings, e.g. according to ISO 12354-2 [1].

The measurement of the dynamic stiffness $s$ is standardized in ISO 9052-1 [2] and DIN EN 29052-1 [3]. One of the experimental setups is shown in Figure 1. The specimen lies on an immobile rigid slab. A rigid plate is placed on top of the specimen and gets excited at its centre. The specimen and the plate above it have a quadratic geometry

*Corresponding author: volker . wittstock@ptb.de with an edge length of $(200 \pm 3) \mathrm{mm}$. This setup realizes the depicted damped oscillator with top excitation. The quantity of interest is the spring of this system.

The measurement procedure scans for the resonance frequency $f_{\mathrm{r}}$ of this system. The apparent dynamic stiffness of the test $s_{\mathrm{t}}^{\prime}$ is calculated from this $f_{\mathrm{r}}$ and the mass per unit area $m_{\mathrm{t}}^{\prime}$ of the upper plate of the test site:

$$
s_{\mathrm{t}}^{\prime}=\left(2 \pi f_{\mathrm{r}}\right)^{2} m_{\mathrm{t}}^{\prime} \text {. }
$$

The standards [2] and [3] then calculate the dynamic stiffness $s$ depending mainly on three ranges of the in-plane flow resistivity $\sigma$ which is measured according to ISO 9053 [4]:

$$
\begin{aligned}
& s^{\prime}=s_{\mathrm{t}}^{\prime} \Longleftrightarrow \sigma \geq 100 \mathrm{kPas} / \mathrm{m}^{2}, \\
& s^{\prime}=s_{\mathrm{t}}^{\prime}+s_{\mathrm{a}}^{\prime} \Longleftrightarrow 10 \mathrm{kPas} / \mathrm{m}^{2} \leq \sigma<100 \mathrm{kPas} / \mathrm{m}^{2}, \\
& s^{\prime}=s_{\mathrm{t}}^{\prime} \Longleftrightarrow \sigma<10 \mathrm{kPas} / \mathrm{m}^{2} \wedge s_{\mathrm{t}}^{\prime} \gg s_{\mathrm{a}}^{\prime},
\end{aligned}
$$

with $s_{\mathrm{a}}^{\prime}$ being the stiffness of the resting air inside the specimen. Instead of (3), the German preamble in [3] uses:

$$
s^{\prime}=s_{\mathrm{t}}^{\prime} \Longleftrightarrow \sigma \geq 1000 \mathrm{kPas} / \mathrm{m}^{2} .
$$

In [5], Kraak discussed the measurement procedure with respect to assumed rigid body motion of the floating floor and the in-plane flow resistivity $\sigma$. Kraak worked out a continuous formula connecting $s_{\mathrm{t}}^{\prime}$ and $s^{\prime}$ under measurement conditions. Hypothetically because of that paper, 


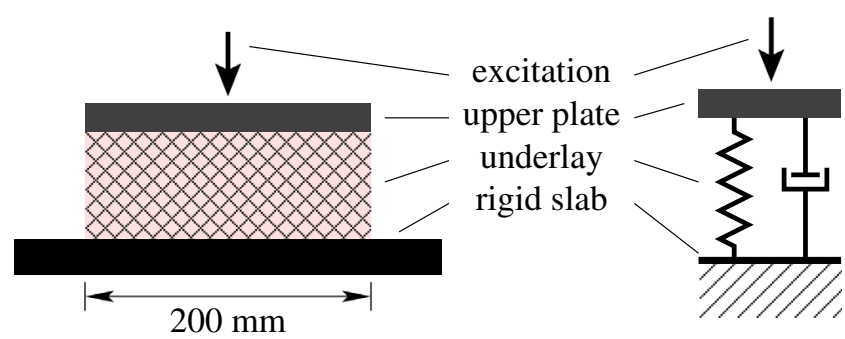

Figure 1. Schematic setup (left) and mechanical analogy (right) of an experimental setup according to [2].

the standards [2] and [3] calculate $s^{\prime}$ by the ranges (3), (4), (5) and (6) which form asymptotic approximations of Kraak's continuous formula. This will be presented in Section 2 and further discussed in Sections 4-6. It will lead to a suggestion for a revision of the measurement procedure of [2] and [3] with respect to the handling of the in-plane flow resistivity $\sigma$.

In [6], the influence of static load and thereby of the density on the flow resistivity was examined. It was shown experimentally that this can shift the flow resistivity of a given material among the above mentioned ranges (3), (4), and (5).

To circumvent a calculation and instead gain the dynamic stiffness of the later application directly from measurements, [7] imposed more real boundary conditions to the specimen. The material under test was taken in larger samples of up to $1000 \mathrm{~mm}$ edge length. As in the standard [2], only a separate centre section of $200 \mathrm{~mm}$ edge length was subject to excitation and measurement. The results were compared to the standardized measurements with open and closed boundaries.

While the standard [2] defines sinusoidal, impact or white noise signals for excitation, [8] also tried exponential swept sine and maximum length sequences and compared them with respect to experimental applicability. Further details about the measurement procedure that are not explicitly standardized in [2] were also discussed, including the read-out of the resonance frequency $f_{\mathrm{r}}$ and the extrapolation due to nonlinear properties of materials.

Relevant factors for sound transmission by floating floors are listed in [9].

Measurement (2) and prediction (1) are both based on a simple oscillator model with uniform motion of a rigid upper plate. For real plates and real lateral dimensions, local motion by bending in the vicinity of the excitation is expected. This paper therefore extends Kraak's theory [5] to flexible upper plates in Section 3 and validates this in Sections 4-6. In Section 7, lateral dimensions of typical room size are examined.

Real materials usually exhibit a nonlinear behaviour and therefore must be tested in a realistic working point using an extrapolation to zero excitation - effectively resulting in a linearization around that working point. To achieve that working point, the upper plate is standardized to exert a realistic load by having a mass of $(8 \pm 0.5) \mathrm{kg}$. In this paper, all mathematical systems are linear and, consequently, free of the above restrictions.
The applied material parameters, geometric parameters and further derived quantities are listed in Table 1.

\section{Kraak's calculation}

In his paper [5], Kraak modelled the measurement setup with quadratic and circular geometry. He assumed the upper plate to be rigid. This upper plate is excited harmonically. At the outer boundary, the system is open. For the air inside the underlay, Kraak neglected the inertia compared to the damping, and he assumed isothermal changes. Kraak also assumed that the resulting stiffness of the underlay can be separated into one part from the structure and one part from the air inside that structure.

Because, according to [9], measurements are expected to be performed at frequencies about $70 \mathrm{~Hz}$, all further calculations in this paper are performed using adiabatic changes. For comparability, the calculations of Kraak are presented here with adiabatic instead of isothermal changes. Results differ only in the adiabatic exponent $\kappa$, which would simply be absent in Kraak's original isothermal case. Also, the solutions for a closed outer boundary (airtight termination) are explicitly provided here.

Kraak derived the following equation for the complex amplitude $\hat{p}$ of the pressure of the air inside the underlay. $\Delta$ is the Laplacian. The values of the used quantities are given in Table 1:

$$
\Delta \hat{p}-\mathrm{i} \alpha^{2} \hat{p}=-\frac{\mathrm{i} \omega \sigma}{d} \hat{w}_{0} .
$$

For circular geometry with radial coordinate $r$, this yields Bessel's differential equation of zero order and an according solution with Bessel functions $J_{0}$ and $Y_{0}$ and a constant particular solution:

$$
\hat{p}(r)=C_{1} J_{0}(\sqrt{-\mathrm{i}} \alpha r)+C_{2} Y_{0}(\sqrt{-\mathrm{i}} \alpha r)+\frac{\kappa p_{0}}{d \varepsilon} \hat{w}_{0} .
$$

The constants $C_{1}$ and $C_{2}$ are calculated from boundary conditions: Always is $0=\hat{p}^{\prime}(0)$, to realize symmetry at the centre. At the outer boundary, the system can be open via $0=\hat{p}(R)$, or closed via $0=\hat{p}^{\prime}(R)$.

In the open case, Kraak's solution is:

$$
\hat{p}(r)=\frac{\kappa p_{0}}{d \varepsilon} \hat{w}_{0}\left[1-\frac{J_{0}(\sqrt{-\mathrm{i}} \alpha r)}{J_{0}(\sqrt{-\mathrm{i}} \alpha R)}\right] .
$$

In the closed case, the solution is constant in $r$ :

$$
\hat{p}(r)=\frac{\kappa p_{0}}{d \varepsilon} \hat{w}_{0} .
$$

For quadratic geometry with edge length $2 L$ and coordinates $x$ and $y$, each in the range $[-L ;+L]$, the homogeneous Helmholtz equation has to be solved first. This yields the eigenfunctions. Using a linear combination of them, the solution $\hat{p}(x, y)$ is calculated.

The solution of the Helmholtz equation includes the boundary conditions: Again, there has to be symmetry at 
Table 1. Basic quantities and their values.

\begin{tabular}{|c|c|c|c|}
\hline Quantity & Symbol & Value & Unit \\
\hline $\begin{array}{l}\text { Upper plate made of steel } \\
\text { Mass density } \\
\text { Young's modulus } \\
\text { Poisson's ratio } \\
\text { Thickness }\end{array}$ & $\begin{array}{l}\varrho \\
E \\
v \\
h\end{array}$ & $\begin{array}{l}8.0 \times 10^{3} \\
2.0 \times 10^{11} \\
0.33 \\
0.025\end{array}$ & $\begin{array}{l}\mathrm{kg} / \mathrm{m}^{3} \\
\mathrm{~N} / \mathrm{m}^{2} \\
1 \\
\mathrm{~m}\end{array}$ \\
\hline $\begin{array}{l}\text { Upper plate made of concrete } \\
\text { Mass density } \\
\text { Young's modulus } \\
\text { Poisson's ratio } \\
\text { Thickness } \\
\end{array}$ & $\begin{array}{l}\varrho \\
E \\
v \\
h\end{array}$ & $\begin{array}{c}2.0 \times 10^{3} \\
1.0 \times 10^{10} \\
0.33 \\
0.060\end{array}$ & $\begin{array}{l}\mathrm{kg} / \mathrm{m}^{3} \\
\mathrm{~N} / \mathrm{m}^{2} \\
1 \\
\mathrm{~m}\end{array}$ \\
\hline $\begin{array}{l}\text { Underlay of porous material } \\
\text { Porosity } \\
\text { Structure factor } \\
\text { Flow resistivity } \\
\text { Stiffness per unit area of the solid structure } \\
\text { Thickness } \\
\end{array}$ & $\begin{array}{r}\varepsilon \\
\chi \\
\sigma \\
s_{\mathrm{s}}^{\prime} \\
d \\
\end{array}$ & $\begin{array}{l}0.50 \\
1.0 \\
1.0 \times 10^{4} \\
1.0 \times 10^{6} \\
0.020\end{array}$ & $\begin{array}{c}1 \\
1 \\
\mathrm{~Pa} \mathrm{~s} / \mathrm{m}^{2} \\
\mathrm{~Pa} / \mathrm{m} \\
\mathrm{m} \\
\end{array}$ \\
\hline $\begin{array}{l}\text { Overall geometry } \\
\text { Half edge length } \\
\text { Outer radius } \\
\text { Inner radius } \\
\end{array}$ & $\begin{array}{c}L \\
R \\
r_{\text {in }} \\
\end{array}$ & $\begin{array}{c}0.10 \\
0.10 \\
1.0 \times 10^{-3} \\
\end{array}$ & $\begin{array}{l}\mathrm{m} \\
\mathrm{m} \\
\mathrm{m}\end{array}$ \\
\hline $\begin{array}{l}\text { Harmonic excitation at the centre of the upper } \\
\text { Angular frequency } \\
\text { Force amplitude } \\
\text { Deflection amplitude }\end{array}$ & $\begin{array}{l}\omega \\
\hat{F}_{0} \\
\hat{w}_{0}\end{array}$ & $\begin{array}{l}\text { Varies } \\
\text { Varies, complex } \\
\text { Varies, complex }\end{array}$ & $\begin{array}{l}1 / \mathrm{s} \\
\mathrm{N} \\
\mathrm{m}\end{array}$ \\
\hline $\begin{array}{l}\text { Derived quantities } \\
\text { Mass per unit area of the upper plate } \\
\text { Bending stiffness of the upper plate }\end{array}$ & $\begin{array}{r}m_{\mathrm{t}}^{\prime} \\
B\end{array}$ & $\frac{\varrho h}{\frac{E h^{3}}{12\left(1-v^{2}\right)}}$ & $\begin{array}{c}\mathrm{kg} / \mathrm{m}^{2} \\
\mathrm{Nm}\end{array}$ \\
\hline Imaginary unit & $\mathrm{i}$ & $\sqrt{-1}$ & 1 \\
\hline
\end{tabular}

the centre via $0=\left.\frac{\partial \hat{p}}{\partial x}\right|_{x=0}=\left.\frac{\partial \hat{p}}{\partial y}\right|_{y=0}$. At the outer boundary, the system can be open via $0=\left.\hat{p}\right|_{x=L}=\left.\hat{p}\right|_{y=L}$, or closed via $0=\left.\frac{\partial \hat{p}}{\partial x}\right|_{x=L}=\left.\frac{\partial \hat{p}}{\partial y}\right|_{y=L}$.

In the open case, Kraak's solution is:

$$
\hat{p}(x, y)=\sum_{n, m=0}^{\infty} c_{n m} \cos k_{x} x \cos k_{y} y,
$$

with coefficients:

$$
c_{n m}=\frac{4}{L^{2}} \frac{\mathrm{i} \omega \sigma}{d} \hat{w}_{0} \frac{(-1)^{n+m}}{k_{x} k_{y}\left[k_{x}^{2}+k_{y}^{2}+\mathrm{i} \alpha^{2}\right]},
$$

and with eigenvalues:

$$
k_{x}=\frac{\pi}{L} \frac{2 n+1}{2}, \quad k_{y}=\frac{\pi}{L} \frac{2 m+1}{2} .
$$

In the closed case, all coefficients except $c_{00}$ are zero. The solution is constant in $x$ and $y$ :

$$
\hat{p}(x, y)=\frac{\kappa p_{0}}{d \varepsilon} \hat{w}_{0} .
$$

The velocity of the air flow inside the underlay is calculated from the gradient of $\hat{p}$. In the closed cases (10) and (14), the constant solutions of $\hat{p}$ yield no air flow. Therefore, the factor of proportionality between $\hat{p}$ and $\hat{w}_{0}$ in (10) and (14) is the dynamic stiffness per unit area of the resting air inside the underlay:

$$
s_{\mathrm{a}}^{\prime}=\frac{\kappa p_{0}}{d \varepsilon} .
$$

This formula is also given in [2] and [3], but without $\kappa$ because of isothermal changes.

In the open cases, Kraak deduced the dynamic stiffness per unit area $s_{\sigma}^{\prime}$ of the flowing air inside the underlay by the 
spatial average $\overline{\hat{p}}$ of the pressure referred to the amplitude $\hat{w}_{0}$ of the excitation. As only the stiffness (the storage modulus) is of interest and not the damping (the loss modulus), Kraak used the real part of the emerging quantity:

$$
s_{\sigma}^{\prime}=\operatorname{Re}\left[\frac{\overline{\hat{p}}}{\hat{w}_{0}}\right] .
$$

In the circular case, this yields:

$$
s_{\sigma}^{\prime}=\frac{\kappa p_{0}}{d \varepsilon} \operatorname{Re}\left[1-\frac{2}{z} \frac{J_{1}(z)}{J_{0}(z)}\right]_{z=\sqrt{-\mathrm{i}}(\alpha R)} .
$$

In the quadratic case, it is:

\section{See equation (18) at the bottom of the page}

Concerning the convergence of the infinite sum in (18), the second factor has the form:

$$
\frac{1}{1-\mathrm{i}\left(\frac{x_{0}}{z}\right)^{2}}=\frac{z^{4}}{z^{4}+x_{0}^{4}}+\mathrm{i}\left[\frac{z^{2}}{x_{0}^{2}}+\frac{x_{0}^{2}}{z^{2}}\right]^{-1},
$$

with real valued positive quantities $x_{0}$ and $z$. Real and imaginary parts both remain in the interval $[0 ; 1]$ and converge to zero when $x_{0}$ rises. As a result, the convergence of (18) is dominated by the first factor of the sum. A break criterion $n, m=20$ was chosen and numerical tests confirmed sufficient convergence.

As mentioned, Kraak assumed that the resulting stiffness $s_{\mathrm{t}}^{\prime}$ of the measurement can be separated into one part from the structure and one part from the air inside that structure. The former part is $s_{\mathrm{s}}^{\prime}$. And because all standardized measurement setups of [2] implement an open outer boundary, the latter part is $s_{\sigma}^{\prime}$ :

$$
s_{\mathrm{t}}^{\prime}=s_{\mathrm{s}}^{\prime}+s_{\sigma}^{\prime} .
$$

The requested dynamic stiffness $s^{\prime}$ is defined as the sum of the stiffness of the solid structure $s_{\mathrm{s}}^{\prime}$ plus the stiffness of the resting air $s_{\mathrm{a}}^{\prime}$ inside that structure:

$$
s^{\prime}=s_{\mathrm{s}}^{\prime}+s_{\mathrm{a}}^{\prime} .
$$

Refering to resting air is, within Kraak's model, only possible with a closed outer boundary. This turns $s^{\prime}$ into an abstract value that is useful for comparing different materials. It does not claim to represent boundary conditions of real rooms - although it might come close as shown below.

This meaning of $s^{\prime}$ here differs from [7], where $s^{\prime}$ is meant to be measured under boundary conditions of real rooms using a modified setup.

Now, $s^{\prime}$ can be calculated from (20) and (21) using (15) and (17) or (18):

$$
s^{\prime}=s_{\mathrm{t}}^{\prime}+s_{\mathrm{a}}^{\prime}-s_{\sigma}^{\prime}=s_{\mathrm{t}}^{\prime}+s_{\mathrm{a}}^{\prime}\left[1-\frac{s_{\sigma}^{\prime}}{s_{\mathrm{a}}^{\prime}}\right] .
$$

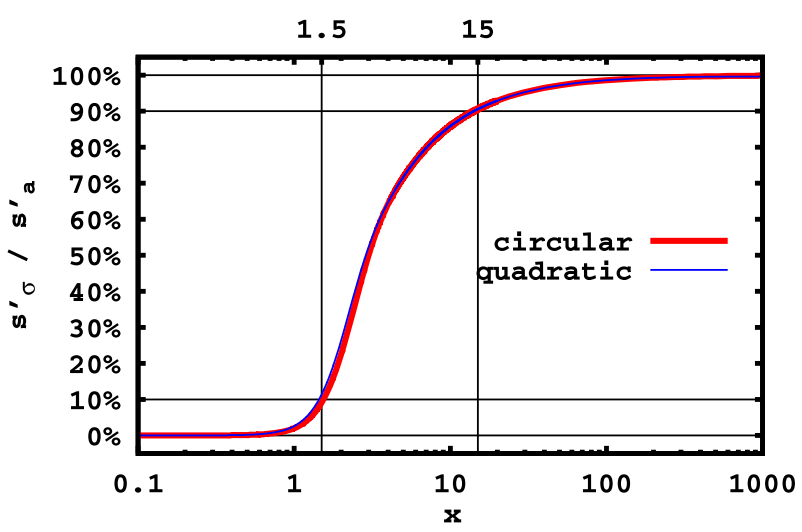

Figure 2. Ratio of the stiffnesses per unit area of flowing and resting air $s_{\sigma}^{\prime} / s_{\mathrm{a}}^{\prime}$ over $x=\alpha R$ (circular) or $x=\alpha L$ (quadratic), plus marker lines.

Kraak examined the ratio $s_{\sigma}^{\prime} / s_{\mathrm{a}}^{\prime}$. This is shown in Figure 2, using the dimensionless quantity $x=\alpha R$ in the circular case and $x=\alpha L$ in the quadratic case. The graphs for circular and quadratic geometry are nearly identical.

For large lateral dimensions $R$ or $L$, like in real rooms, and for suitable $\alpha$, both graphs approach $100 \%$. This means $s_{\sigma}^{\prime} \approx s_{\mathrm{a}}^{\prime}$, i.e. the air comes to rest and the system becomes equivalent to the closed case. This indicates that (21) might be an approximation for situations in real rooms.

Kraak proposed the two following asymptotic cases based on Figure 2:

Case 1: If $s_{\sigma}^{\prime} / s_{\mathrm{a}}^{\prime}>90 \%$ (for $x>15$ approximately), then Kraak suggests an approximation by $s_{\sigma}^{\prime} / s_{\mathrm{a}}^{\prime} \approx 1$, and $(22)$ simply yields:

$$
s^{\prime} \approx s_{\mathrm{t}}^{\prime}
$$

Case 2: If $s_{\sigma}^{\prime} / s_{\mathrm{a}}^{\prime}<10 \%$ (for $x<1.5$ approximately), then Kraak proposes to use $s_{\sigma}^{\prime} / s_{\mathrm{a}}^{\prime} \approx 0$, and therefore $(22)$ becomes:

$$
s^{\prime} \approx s_{\mathrm{t}}^{\prime}+s_{\mathrm{a}}^{\prime} .
$$

No proposal is given by Kraak for the range in between.

How do these two cases translate to the flow resistivity $\sigma$ and how does this correlate to the ranges (3), (4), (5), and (6) of the standards [2] and [3]? Solving $x=\alpha R$ for $\sigma$ leads to:

$$
\sigma=\frac{x^{2} \kappa p_{0}}{R^{2} \omega \varepsilon} .
$$

With values from Table 1 , with $f \approx 70 \mathrm{~Hz}$ from [9], and with $\kappa=1$ due to the isothermal assumption in [2] and [3], the two cases yield:

$$
s^{\prime} \approx s_{\mathrm{t}}^{\prime} \Longleftrightarrow \sigma>\approx 10000 \mathrm{kPas} / \mathrm{m}^{2},
$$

$$
s_{\sigma}^{\prime}=\frac{\kappa p_{0}}{d \varepsilon} \operatorname{Re}\left[\frac{64}{\pi^{4}} \sum_{n, m=0}^{\infty} \frac{1}{(2 n+1)^{2}(2 m+1)^{2}} \cdot \frac{1}{1-\mathrm{i}\left(\frac{\pi}{z}\right)^{2}\left[(2 n+1)^{2}+(2 m+1)^{2}\right]}\right]_{z=2(\alpha L)} .
$$




$$
s^{\prime} \approx s_{\mathrm{t}}^{\prime}+s_{\mathrm{a}}^{\prime} \Longleftrightarrow \sigma<\approx 100 \mathrm{kPa} \mathrm{s} / \mathrm{m}^{2} .
$$

There is no match between this set of assignments and the standardized set (3), (4), (5), and (6). Especially, the extra condition $s_{\mathrm{t}}^{\prime} \gg s_{\mathrm{a}}^{\prime}$ in (5) cannot be deduced from the presented theory. These ranges are further discussed in Section 5 .

\section{Extending Kraak's approach}

This paper goes beyond the calculation of Kraak [5]. First, the inertia of the air inside the underlay is taken into account and adiabatic changes are assumed, i.e. $\kappa$ is always present. The main difference to [5] is a flexible upper plate that is modelled according to Bernoulli's theory - especially with regard to the room-sized situation.

So far, only the circular geometry with radial coordinate $r$ was treated. A harmonic ansatz with angular frequency $\omega$ is made. Consequently, only complex amplitudes show up. The following differential equations arise for the pressure $\widehat{p}(r)$ and the velocity $\widehat{\underline{v}}(r)$ of the air inside the underlay, and for the deflection $\widehat{w}(r)$ of the upper plate. For values of the parameters see Table 1 :

$$
\begin{aligned}
-\operatorname{grad} \widehat{p}(r) & =\gamma \widehat{\widehat{v}}(r), \\
-\operatorname{div} \underline{\hat{v}}(r) & =\frac{\mathrm{i} \omega}{\kappa p_{0}} \widehat{p}(r)-\frac{\mathrm{i} \omega}{d \varepsilon} \widehat{w}(r), \\
B \Delta \Delta \widehat{w}(r) & =\delta \widehat{w}(r)-\widehat{p}(r) .
\end{aligned}
$$

A velocity potential $\widehat{\Phi}(r)$ is introduced for $\underline{\widehat{v}}(r)$. The pressure $\widehat{p}(r)$ is then calculated from (28). These both mean:

$$
\begin{aligned}
& \widehat{\widehat{v}}(r) \stackrel{\text { Def }}{=}-\operatorname{grad} \widehat{\Phi}(r), \\
& \widehat{p}(r)=\gamma \widehat{\Phi}(r) .
\end{aligned}
$$

The differential equations for $\widehat{w}(r)$ and $\widehat{\Phi}(r)$ finally are:

$$
\begin{aligned}
B \Delta \Delta \widehat{w}(r) & =\delta \widehat{w}(r)-\gamma \widehat{\Phi}(r), \\
\Delta \widehat{\Phi}(r) & =\frac{i \omega \gamma}{\kappa p_{0}} \widehat{\Phi}(r)-\frac{i \omega}{d \varepsilon} \widehat{w}(r) .
\end{aligned}
$$

This is a coupled eigenvalue problem. Bessel's normal and modified differential equations yield the eigenfunctions for the Laplacian in the form of Bessel functions $J_{0}$ and $Y_{0}$ and modified Bessel functions $I_{0}$ and $K_{0}$. The two latter can be expressed by the two former using complex arguments. Due to the coupling, $\widehat{w}(r)$ and $\widehat{\Phi}(r)$ need an equivalent ansatz (c.f. [10], Sect. 58) with coefficient $\lambda$ :

$$
\widehat{w}(r)=\widehat{w}_{1} J_{0}(\lambda r)+\widehat{w}_{2} Y_{0}(\lambda r)+\widehat{w}_{3} J_{0}(\mathrm{i} \lambda r)+\widehat{w}_{4} Y_{0}(\mathrm{i} \lambda r),
$$

$$
\widehat{\Phi}(r)=\widehat{\Phi}_{1} J_{0}(\lambda r)+\widehat{\Phi}_{2} Y_{0}(\lambda r)+\widehat{\Phi}_{3} J_{0}(\mathrm{i} \lambda r)+\widehat{\Phi}_{4} Y_{0}(\mathrm{i} \lambda r) .
$$

This transforms the differential equations (33) and (34) for the functions $\widehat{w}(r)$ and $\widehat{\Phi}(r)$ into algebraic equations for the coefficients $\widehat{w}_{n}$ and $\widehat{\Phi}_{n}$ of (35) and (36). These eight algebraic equations are composed of four independent parts:

$$
\left[\begin{array}{cc}
\delta-\lambda^{4} B & -\gamma \\
-\frac{\mathrm{i} \omega}{d \varepsilon} & \zeta \lambda^{2}+\frac{\mathrm{i} \omega \gamma}{\kappa p_{0}}
\end{array}\right] \cdot\left[\begin{array}{l}
\widehat{w}_{n} \\
\widehat{\Phi}_{n}
\end{array}\right]=\left[\begin{array}{l}
0 \\
0
\end{array}\right],
$$

with $n=1,2,3,4$ and:

$$
\zeta \stackrel{\text { Def }}{=}\left\{\begin{array}{ll}
+1 & \text { for } n=1,2 \\
-1 & \text { for } n=3,4
\end{array} .\right.
$$

Non-trivial solutions of the homogenous system (37) exist only, if the system is singular. The according determinant of the coefficients forms an equation for the eigenvalues $\lambda$ :

$$
0=\lambda^{6} B+\lambda^{4} \zeta B \frac{\mathrm{i} \omega \gamma}{\kappa p_{0}}-\lambda^{2} \delta-\zeta \mathrm{i} \omega \gamma\left[\frac{\delta}{\kappa p_{0}}-\frac{1}{d \varepsilon}\right] .
$$

This is a cubic equation for $\lambda^{2}$. If $\lambda$ is a solution, so is $-\lambda$. Due to the symmetry $J_{0}(z)=J_{0}(-z)$ (same for $Y_{0}$ ), this yields identical solutions. This means, there are only three independent solutions $\lambda_{1}, \lambda_{2}$ and $\lambda_{3}$.

If $\lambda^{2}$ is a solution for $\zeta=+1$, then $-\lambda^{2}$ is a solution for $\zeta=-1$. Therefore, if $\lambda$ is a solution for $n=1,2$, then $\mathrm{i} \lambda$ is a solution for $n=3,4$. Due to $J_{0}(\mathrm{i}(\mathrm{i} \lambda) r)=J_{0}(-\lambda r)=J_{0}(\lambda r)$ (same for $Y_{0}$ ), the branch $n=3$, 4 leads to the same solutions as the branch $n=1,2$. Consequently, the branch $n=3,4$ is no longer needed.

If $\lambda_{1}, \lambda_{2}$ and $\lambda_{3}$ are solutions of (39), $\widehat{w}_{n}$ and $\widehat{\Phi}_{n}$ can be taken e.g. from the first line of (37). This yields:

$$
\left[\begin{array}{c}
\widehat{w}(r) \\
\widehat{\Phi}(r)
\end{array}\right]=\sum_{j=1,2,3}\left[\begin{array}{c}
\gamma \\
\delta-\lambda_{j}^{4} B
\end{array}\right]\left[C_{j} J_{0}\left(\lambda_{j} r\right)+D_{j} Y_{0}\left(\lambda_{j} r\right)\right],
$$

with coefficients $C_{j}$ and $D_{j}$ to be calculated from boundary conditions later.

The coefficients of the $\lambda^{2}$-term and of the constant term in (39) can change their signs, thereby possibly changing some properties of the solutions. This occurs at the frequencies:

$$
\begin{gathered}
f_{1}=\frac{1}{2 \pi} \sqrt{\frac{s_{\mathrm{s}}^{\prime}}{m_{\mathrm{t}}^{\prime}}}, \\
f_{2}=\frac{1}{2 \pi} \sqrt{\frac{s_{\mathrm{s}}^{\prime}+s_{\mathrm{a}}^{\prime}}{m_{\mathrm{t}}^{\prime}}} .
\end{gathered}
$$

The frequency in (41) marks the oscillation of the plate on the stiffness of the structure alone. The frequency in (42) describes this for the combined stiffness of the structure and the resting air. As discussed in [5], $f_{1}$ and $f_{2}$ mark the possible extremes for low and high flow resistivity, respectively.

Only at frequency $f_{2}$, one solution of (39) becomes zero, e.g. $\lambda_{1}$. The according solutions of (33) and (34) then are no 
longer Bessel functions, but elements of the kernel of the Laplacian:

$$
\begin{aligned}
& {\left[\begin{array}{l}
\widehat{w}(r) \\
\widehat{\Phi}(r)
\end{array}\right]=\left[\begin{array}{l}
\gamma \\
\delta
\end{array}\right]\left[C_{1}+D_{1} \ln \frac{r}{r_{0}}\right]} \\
& +\sum_{j=2,3} \gamma\left[\begin{array}{c}
1 \\
\lambda_{j}^{2} \\
\mathrm{i} \omega B \\
\kappa p_{0}
\end{array}\right]\left[C_{j} J_{0}\left(\lambda_{j} r\right)+D_{j} Y_{0}\left(\lambda_{j} r\right)\right],
\end{aligned}
$$

with an arbitrary scaling factor $r_{0}$, e.g. $r_{0}=1 \mathrm{~m}$. The partial solution of $C_{1}$ represents the upper plate without deformation, and the air without flow.

For the boundary conditions, $Y_{0}(0)=-\infty$ must be kept in mind. But not all coefficients $D_{j}$ in (40) can be zero, because in that case several boundary conditions could not be fulfilled. A small inner radius $r_{\text {in }}$ with $0<r_{\text {in }} \ll R$ is needed to formulate all boundary conditions:

$$
\begin{aligned}
0 & =\widehat{w}^{\prime}\left(r_{\text {in }}\right) \quad(\text { symmetry, plate }) \\
0 & =\widehat{p}^{\prime}\left(r_{\text {in }}\right) \quad(\text { symmetry, air }) \\
\widehat{w}_{0} & =\widehat{w}\left(r_{\text {in }}\right) \quad(\text { excitation by displacement }), \\
\frac{-\widehat{F}_{0}}{2 \pi r_{\text {in }}} & =\widehat{Q}_{r}\left(r_{\text {in }}\right) \quad(\text { excitation by force }) \\
0 & =\widehat{Q}_{r}(R) \quad(\text { free boundary, plate }) \\
0 & =\widehat{M}_{r}(R) \quad(\text { free boundary, plate }) \\
0 & =\widehat{p}(R) \quad(\text { open boundary, air }) \\
0 & =\widehat{p}^{\prime}(R) \quad(\text { closed boundary, air })
\end{aligned}
$$

with the shear force $\widehat{Q}_{r}$ and the torque $\widehat{M}_{r}$ of the upper plate, both per unit length in circumferential direction (c.f. [10], Sect. 15 and [11], Sect. 16):

$$
\begin{aligned}
& \widehat{M}_{r}(r)=-B\left[\frac{\partial^{2}}{\partial r^{2}}+\frac{v}{r} \frac{\partial}{\partial r}\right] \widehat{w}(r), \\
& \widehat{Q}_{r}(r)=-B \frac{\partial}{\partial r}\left[\frac{\partial^{2}}{\partial r^{2}}+\frac{1}{r} \frac{\partial}{\partial r}\right] \widehat{w}(r) .
\end{aligned}
$$

Of course, only one condition of (50) and (51) can be chosen at a given time. Similarly, only one excitation of (46) and (47) is possible. The respective other quantity of $\widehat{w}_{0}$ and $\widehat{F}_{0}$ is post-calculated.

At $f_{2}$ and with closed boundary (51), only excitation by displacement (46) yields a regular system of boundary conditions. That resonance at $f_{2}$ is without damping. Therefore, $\widehat{F}_{0}$ must be zero. The solution is constant:

$$
\left[\begin{array}{c}
\widehat{w}(r) \\
\widehat{\Phi}(r)
\end{array}\right]=\left[\begin{array}{c}
1 \\
\delta / \gamma
\end{array}\right] \widehat{w}_{0} \text {. }
$$

From the six boundary conditions, the six coefficients $C_{j}$ and $D_{j}$ with $j=1,2,3$ from (40) or (43) can now be calculated. As this $6 \times 6$ matrix is often badly conditioned, the numerical calculation must use numbers with high accuracy. The gmp-library [12] was used.

Calculating a spatial average of the stiffness like Kraak in [5] is not possible here. Should the average of the ratios $\overline{\widehat{p}} / \widehat{w}$ be used or the ratio of the averages $\overline{\hat{p}} / \overline{\widehat{w}}$ ? Both variants yield strange results. Instead, the admittance $Y$ at the point of the excitation and its level $L_{Y}$ are used as the comparing quantities:

$$
\begin{gathered}
Y=\mathrm{i} \omega \widehat{w}_{0} / \widehat{F}_{0}, \\
L_{Y}=10 \log _{10} \frac{|Y|}{Y_{\text {ref }}} \mathrm{dB}, \quad Y_{\text {ref }} \stackrel{\text { Def }}{=} \frac{1}{400} \frac{\mathrm{m}}{\mathrm{s} \mathrm{N}} .
\end{gathered}
$$

\section{Simulations with standard setup}

The standard [2] requires a quadratic upper plate with an edge length of $(200 \pm 3) \mathrm{mm}$ and a mass of $(8 \pm 0.5)$ $\mathrm{kg}$. For the flexible upper plates, only the circular geometry has been calculated so far. To replace the aforementioned standardized quadratic plate by a circular one, the mass per unit area $m_{\mathrm{t}}^{\prime}$ and the eigenfrequencies should be kept unchanged or at least as similar as possible. Concerning $m_{\mathrm{t}}^{\prime}$, the same material parameters and the same thickness were chosen. Concerning the eigenfrequencies, similar lateral dimensions were set: $R=L$.

The upcomming calculations have been performed with the parameters from Table 1 with an upper plate made of steel. This yields the critical frequencies $f_{1} \approx 11.3 \mathrm{~Hz}$ and $f_{2} \approx 43.9 \mathrm{~Hz}$ after (41) and (42). For comparison, Kraak's solution (with adiabatic changes as discussed in Sect. 2) is included in most figures.

First, the calculations by Kraak and the authors are compared concerning their admittance $Y$ at the point of excitation. In this case, both types of boundary conditions (50) and (51) were applied. Both sets of calculations yield the same results as shown in Figure 3.

The chosen flow resistivity $\sigma$ from Table 1 is a low value according to the discussion at the end of Section 2. The air flow therefore is hardly obstructed. In the open case, the air can freely leave the underlay and consequently does not add significantly to the overall stiffness of the system. The resonance in this open case is found at frequency $f_{1}$, as expected by (41). In the closed case, the air cannot leave the system. The air does not flow and acts as an additional spring. The resonance is at $f_{2}$ from (42).

The upcomming calculations have been performed with an open outer boundary according to (50). The closed case of equation (51) is discussed in detail in Section 6.

Next, the flow resistivity $\sigma$ was varied. Results are shown in Figure 4. The values for $\sigma$ are given in the legend.

The first three curves (black, gold, red), corresponding to low values of $\sigma$, are centred around frequency $f_{1}$ and widen successively. Increasing the flow resistivity causes increased damping. But the air can still leave the underlay so easily, that it hardly contributes to the overall stiffness. 


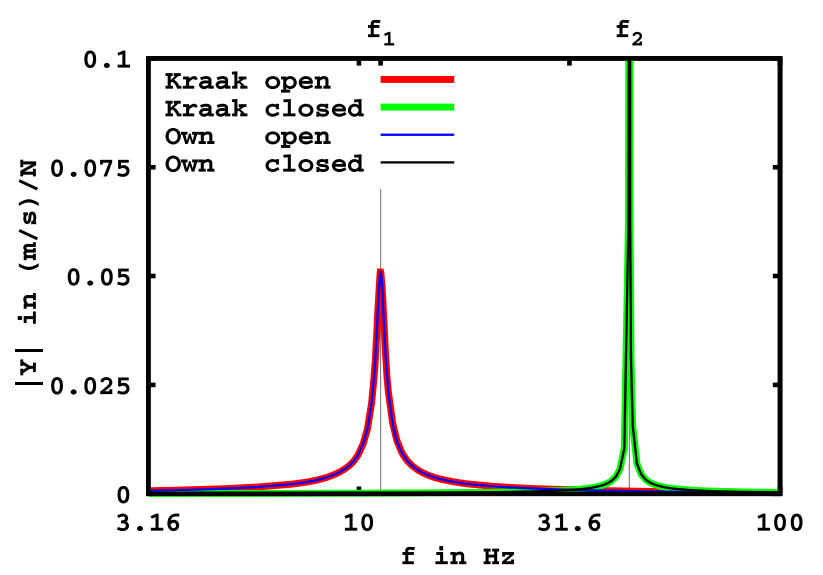

Figure 3. Magnitude of the admittance at the point of excitation. Comparison between Kraak's and own calculations, using open and closed outer boundary conditions according to (50) and (51) respectively.

The next two curves (cyan, magenta) continue to widen up as the damping increases and the resonance frequency $f_{\mathrm{r}}$ moves from $f_{1}$ towards $f_{2}$. This is because the airflow becomes increasingly obstructed, preventing the air from leaving the underlay, thereby adding to the stiffness of the system. The system has maximum damping.

The resonance frequencies $f_{\mathrm{r}}$ of the three final curves (blue, gray, green) approach $f_{2}$. Additionally, the curves slim again significantly. The high flow resistivity finally prevents the airflow. Without flow, there is no damping. The stiffness of the nearly static air approaches $s_{\mathrm{a}}^{\prime}$.

The shift of the resonance frequency $f_{\mathrm{r}}$ depending on the flow resistivity $\sigma$ from Figure 4 is shown in more detail in Figure 5. Kraak's solution is also shown for comparison and both curves agree to a great extent for this set of parameters. Also, Kraak's solution for quadratic geometry is shown exemplarily. There is a small shift between these two groups of curves. The curves are similar for circular and quadratic geometry. All curves cover the range between $f_{1}$ and $f_{2}$.

To examine which system parameters have a relevant impact on $f_{\mathrm{r}}$, the following parameters were varied: the stiffness per unit area of the structure $s_{\mathrm{s}}^{\prime}$, the thickness $d$ and the porosity $\varepsilon$. All associated figures show the curves of the resonance frequency $f_{\mathrm{r}}$ depending on the flow resistivity $\sigma$ for each varied parameter. The red curve from Figure 5, representing the values from Table 1, is always repeated for reference.

Figure 6 shows the influence of the stiffness per unit area of the structure $s_{\mathrm{s}}^{\prime}$ on the resonance frequency $f_{\mathrm{r}}$. The values of $s_{\mathrm{s}}^{\prime}$ are given in the legend. Kraak's solution is shown for comparison and both calculations show very good agreement. For the blue curve, the stiffness of the structure $s_{\mathrm{s}}^{\prime}$ approaches the stiffness of the resting air of $s_{\mathrm{a}}^{\prime} \approx 1.4 \times$ $10^{7} \mathrm{~Pa} / \mathrm{m}$ (for adiabatic changes). If the stiffness of the structure $s_{\mathrm{s}}^{\prime}$ is further increased, the critical frequencies $f_{1}$ and $f_{2}$ also increase, approaching each other until they form a narrow intervall. In that case, the air inside the underlay loses its influence on the system almost completely.

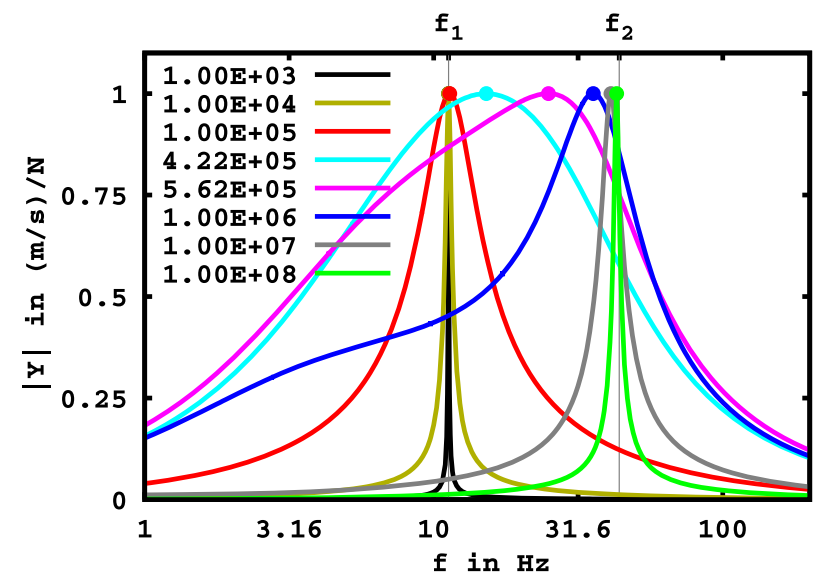

Figure 4. Magnitude of the admittance at the point of excitation. Comparison of different flow resistivities $\sigma$ (unit: $\mathrm{Pa} \mathrm{s} / \mathrm{m}^{2}$ ), scaled to achieve a value of 1 at the maximum, which is emphasized by dots.

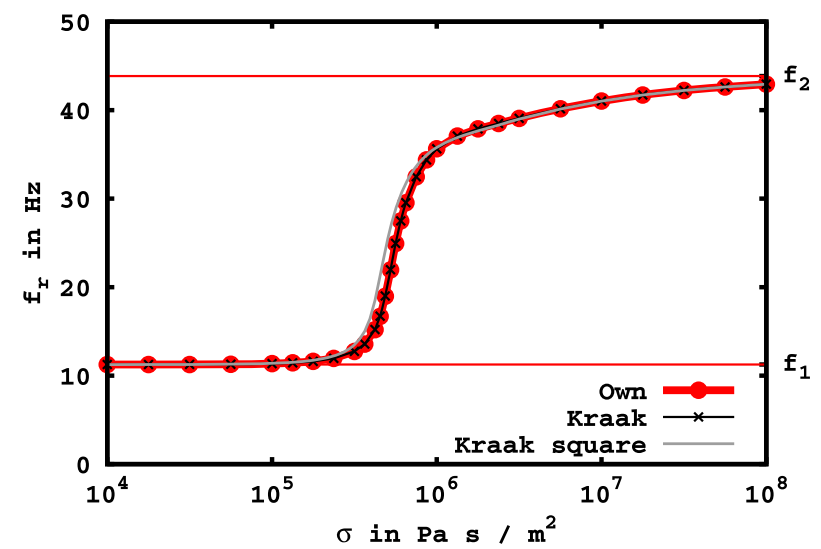

Figure 5. Shift of the resonance frequency $f_{\mathrm{r}}$ depending on the flow resistivity $\sigma$, lines for asymptotes at $f_{1}$ and $f_{2}$.

Figures 7 and 8 accordingly show the variation of the thickness $d$ and the porosity $\varepsilon$. The values of these parameters are given in the respective legends. Kraak's calculation is shown for comparison. The agreement is again very good.

The variation of the structure factor $\chi$ was calculated, too. There was only a minimal difference between curves for $\chi=1$ and $\chi=100$, so the figure is omitted.

\section{Standard evaluation procedure}

Now the evaluation of the dynamic stiffness per unit area $s^{\prime}$ according to [2] and [3] is performed. The different cases with respect to $\sigma$ have already been given in (3), (4), (5) and (6). In the simulations, the exact value of $s^{\prime}$ is a known parameter and can be compared to the output of the evaluation procedure.

The curve from Figure 5 with the given basic parameters from Table 1 is treated first. The result is shown in Figure 9. Some remarks concerning that figure follow: 


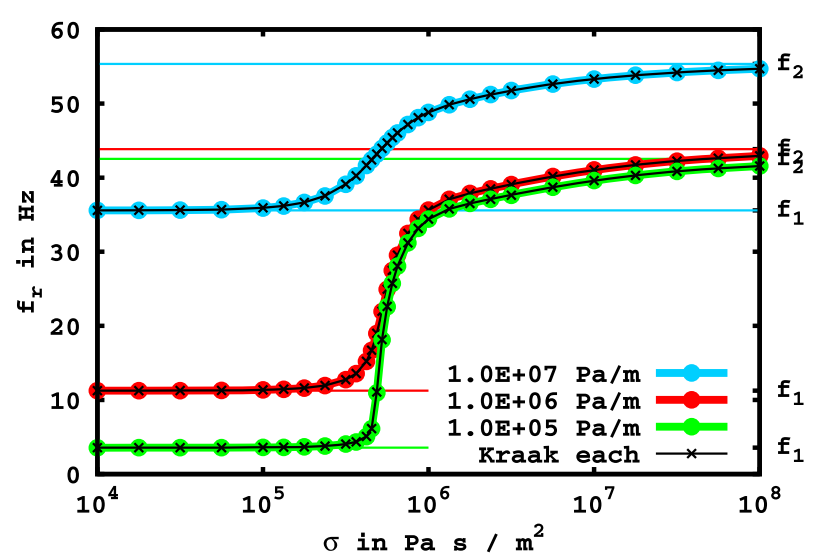

Figure 6. Resonance frequencies $f_{\mathrm{r}}$ for different values of the stiffness per unit area of the solid structure $s_{\mathrm{s}}^{\prime}$ according to the legend, Kraak's respective solutions for comparison, lines for asymptotes at $f_{1}$ and $f_{2}$ in according colors.

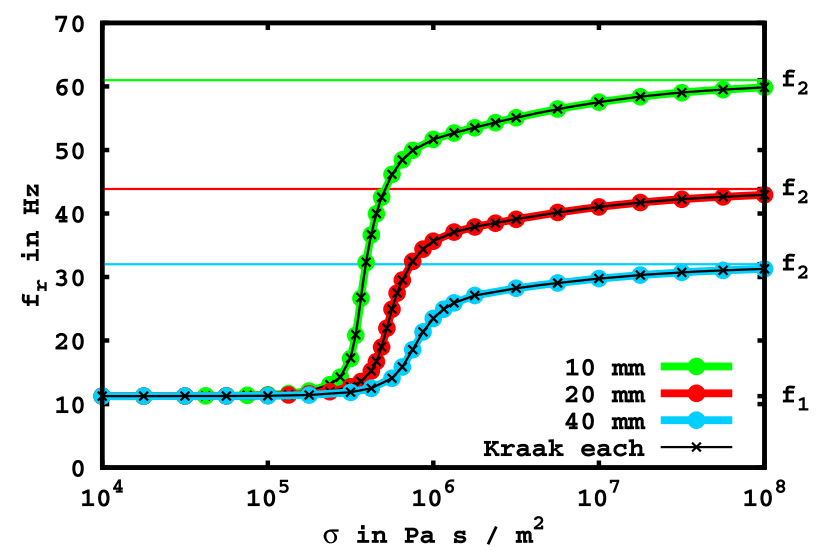

Figure 7. Resonance frequencies $f_{\mathrm{r}}$ for different values of the thickness $d$ according to the legend, Kraak's respective solutions for comparison, lines for asymptotes at $f_{2}$ in according colors.

1. The exact value of $s^{\prime}$ is given by (21). The corresponding curve is labelled exact. It has the same color as the following curve.

2. Evaluation according to the standard procedure of [2] is performed by (3), (4) and (5). The curve is labelled standard and features a significant jump discontinuity at $\sigma=100 \mathrm{kPa} \mathrm{s} / \mathrm{m}^{2}$. This is where the two evaluation procedures are switched.

3. Evaluation according to the German preamble of [3] is performed and shown with label $\mathrm{DE}$. The curve has a gap between $\sigma=100 \mathrm{kPa} \mathrm{s} / \mathrm{m}^{2}$ and $\sigma=$ $1000 \mathrm{kPa} \mathrm{s} / \mathrm{m}^{2}$ where no evaluation is defined.

4. Kraak's correction according to (22) is calculated. The curve is simply labelled Kraak.

5. Exemplary, Kraak's correction for the quadratic case is added. That curve is labelled Kraak square.

For low flow resistivities, the exact value of the dynamic stiffness $s^{\prime}$ is met. When the standard procedure switches its evaluation formula at $\sigma=100 \mathrm{kPa} \mathrm{s} / \mathrm{m}^{2}$, the dynamic stiffness $s^{\prime}$ is at first underestimated by more than one

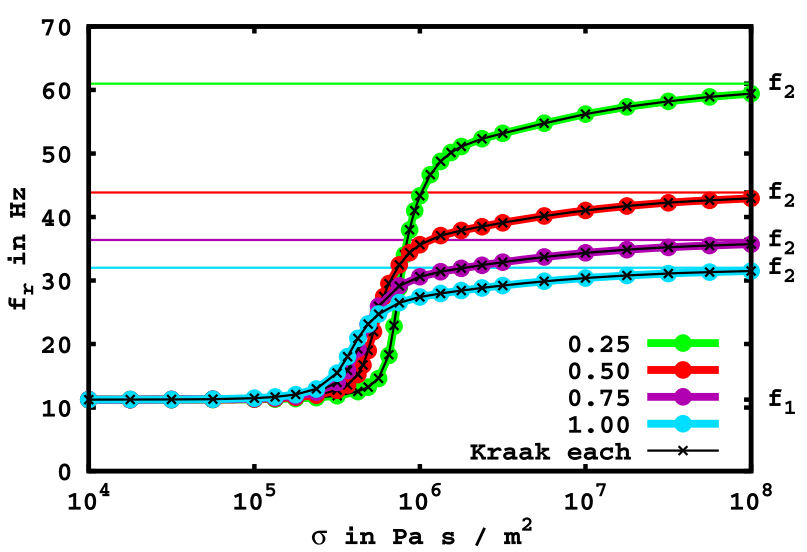

Figure 8. Resonance frequencies $f_{\mathrm{r}}$ for different values of the porosity $\varepsilon$ according to the legend, Kraak's respective solutions for comparison, lines for asymptotes at $f_{2}$ in according colors.

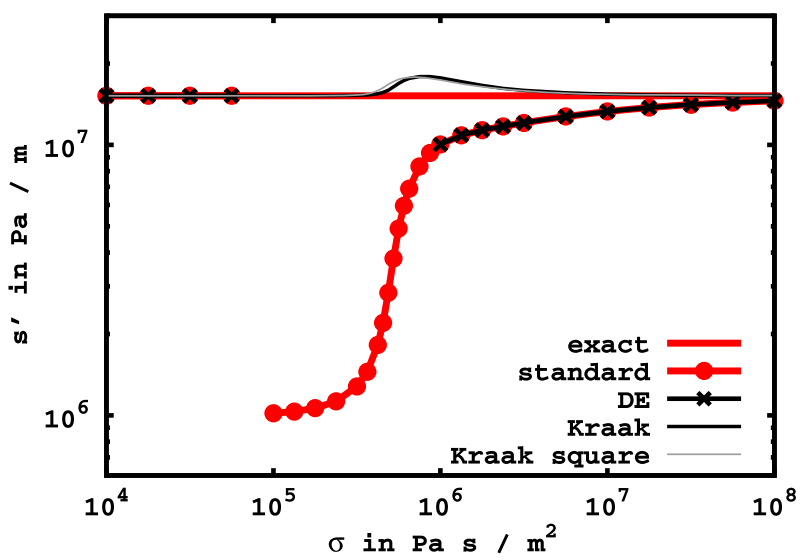

Figure 9. Dynamic stiffness per unit area $s^{\prime}$ : the exact value, the calculation according to [2] using the standard procedure, the calculation according to the German preamble of [3] (DE), and calculation according to Kraak (22) for circular and quadratic geometry.

decade. At $\sigma=1000 \mathrm{kPa} \mathrm{s} / \mathrm{m}^{2}$ a great part of this mismatch has subsided. At this point, the evaluation according to the German preamble sets in again. Thereafter, both evaluations approach the exact value asymptotically. In contrast, Kraak's correction always stays close to the exact value.

The same evaluation has been performed for the parameter variations of the Figures 6,7 and 8 . The results are shown in Figure 10 for the stiffness of the structure $s_{\mathrm{s}}^{\prime}$, in Figure 11 for the thickness $d$, and in Figure 12 for the porosity $\varepsilon$. The figure for the structure factor $\chi$ is omitted again because there are no significant changes visible.

The curves in these figures all show the same general behaviour as those in Figure 9. Especially remarkable are the curves in Figure 10. When the stiffness of the structure $s_{\mathrm{s}}^{\prime}$ is varied, the stiffness of the air gains or looses influence on the system. For a very low $s_{\mathrm{s}}^{\prime}$ (green curve), the standard evaluation procedure of [2] has a jump discontinuity for a 


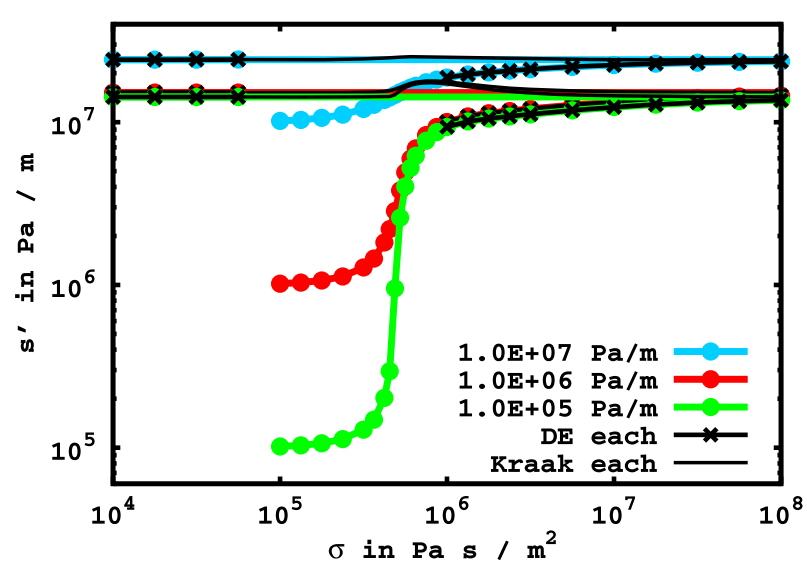

Figure 10. Dynamic stiffness per unit area $s^{\prime}$ for different values of the stiffness per unit area of the solid structure $s_{\mathrm{s}}^{\prime}$ according to the legend: the exact value, the calculation according to [2] using the standard procedure, the calculation according to the German preamble of [3] (DE), and calculation according to Kraak (22).

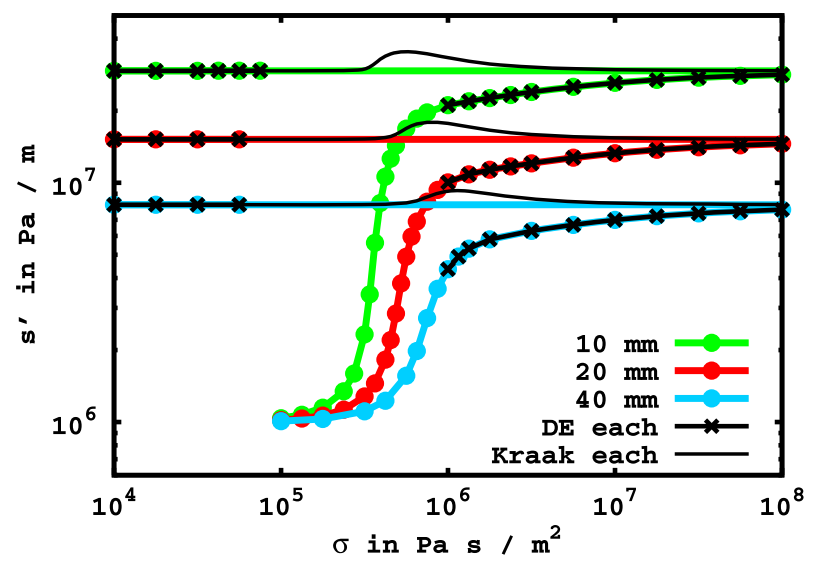

Figure 11. Dynamic stiffness per unit area $s^{\prime}$ for different values of the thickness $d$ according to the legend: the exact value, the calculation according to [2] using the standard procedure, the calculation according to the German preamble of [3] (DE), and calculation according to Kraak (22).

factor more than 100 and, thereby, underestimates the dynamic stiffness significantly! The evaluation according to the German preamble of [3] skips this part with the largest deviations and shows an underestimation factor of about 2, depending on the parameter that was varied. In all cases, the correction according to Kraak yields a result close to the exact value.

This leads to the following assessment of the previously presented evaluation procedures:

- The standard procedure of [2] provides an uninterrupted scheme, but at the expense of significantly underestimating the results of $s^{\prime}$ throughout at least one decade of $\sigma$.

- The procedure of the German preamble of [3] provides results that underestimate the exact value of $s^{\prime}$ only by a factor of about 2 . But due to the gap in its definition, it fails to yield results in a significant range of $\sigma$.

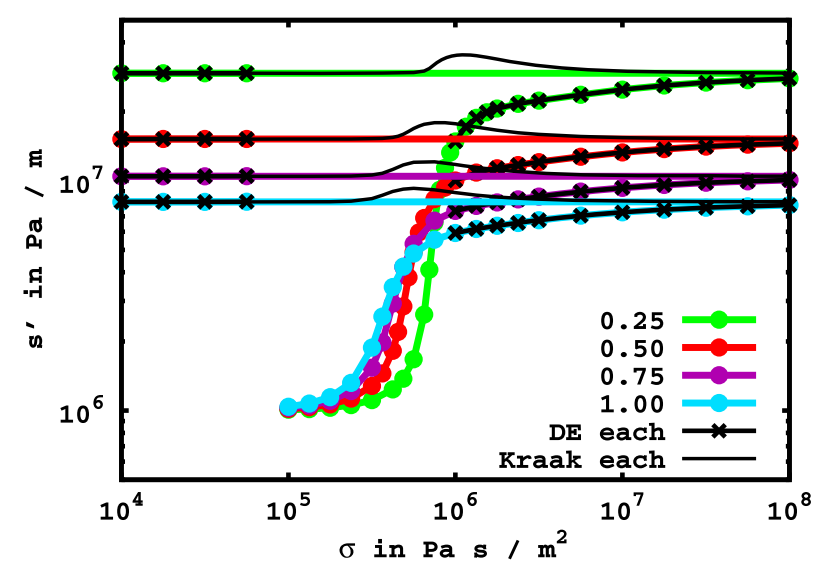

Figure 12. Dynamic stiffness per unit area $s^{\prime}$ for different values of the porosity $\varepsilon$ according to the legend: the exact value, the calculation according to [2] using the standard procedure, the calculation according to the German preamble of [3] (DE), and calculation according to Kraak (22).

- The continuous correction formula of Kraak presents results of $s^{\prime}$ close to the exact value and throughout the complete range of $\sigma$.

Considering all this, the authors favour Kraak's method of correction. The correction curve from Figure 2 is suggested to be added in analytical or tabular form to a revised version of ISO 9052-1 [2].

\section{Closed boundary}

Now the closed boundary condition of (51) is applied instead of (50). For the initial parameters from Table 1, the shift of the resonance frequency $f_{\mathrm{r}}$ like in Figure 5 was calculated. This time, no shift was observed. $f_{\mathrm{r}}$ constantly remains at the critical frequency $f_{2}$ from (42).

The variations of the parameters $s_{\mathrm{s}}^{\prime}, d, \varepsilon$ and $\chi$ from Section 4 have been repeated for the closed boundary condition. Like the initial parameters before, no shift in the resonance frequency occurs. $f_{\mathrm{r}}$ always sticks to the respective value of $f_{2}$.

In all cases, the dynamic stiffness $s^{\prime}$ applied in the calculation could exactly be re-calculated from the simulations using (2). No further correction was needed.

The setup with closed boundary and with that simple evaluation procedure seems to pose a different option for a revision of [2].

To examine the experimental feasibility of this proposal, measurements have been performed with porous materials made from renewables. For the realization of the closed boundaries, a setup with adjustable walls was assembled as shown in Figure 13. The sealing between the enclosing walls and the specimen and the upper plate required remarkable effort.

Open and closed outer boundaries have both been realized in the experiments. In case of the open boundary, the 


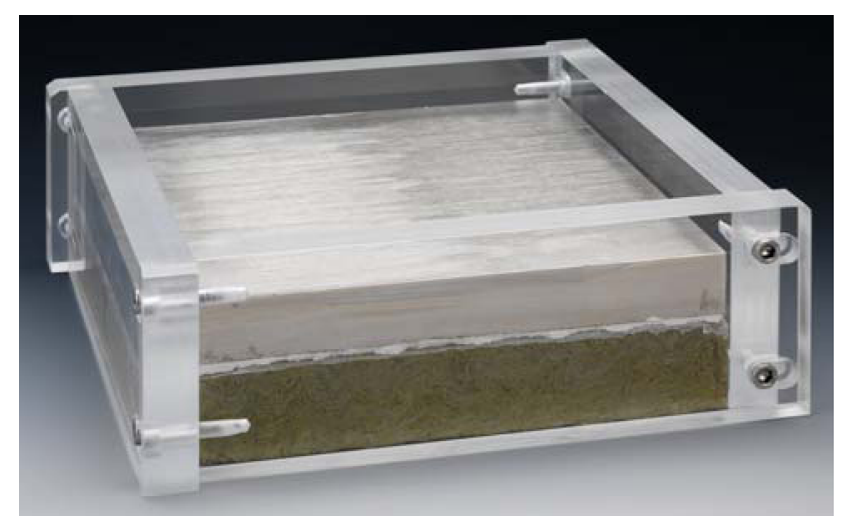

Figure 13. Experimental setup realizing the closed boundary condition (51), using geometrically adjustable walls of acrylic glass.

correction according to Kraak using (22) was applied. Figure 14 shows a comparison of these two variants.

Clearly, the variant with closed boundary yields smaller results most of the time. This is attributed to the experimental problem to realize a tight but non-disturbing connection between the outer walls and the upper plate and the underlay. There remains some leakage. The closed boundary condition cannot be completely achieved in the experimental setup.

These experimental results so far discourage from the implementation of the closed boundary condition in a revision of ISO 9052-1 [2].

\section{Simulations with room setup}

So far in this paper, a circular measurement setup with radius $R=100 \mathrm{~mm}$ and an upper plate made of steel has been discussed.

To approach a real room situation, the upper plate is changed from steel to concrete in a first step. Its values are given in Table 1 (upper plate made of concrete). This changes the critical frequencies from (41) and (42) to $f_{1} \approx 14.5 \mathrm{~Hz}$ and $f_{2} \approx 56.6 \mathrm{~Hz}$. Figure 15 compares the shift of the resonance frequency $f_{\mathrm{r}}$ depending on the flow resistivity $\sigma$ for steel and concrete plates. The red curve is cited from Figure 5 again. Kraak's solution is calculated for comparison - again with very good agreement.

In a second step, the radius $R$ of the setup is increased approaching typical sizes of real rooms. Open boundary conditions according to (50) were used, unless noted otherwise. Basic values from Table 1 are applied.

By increasing radius $R$, the eigenfrequencies of the system shift to lower frequencies. They enter the measurement region between $f_{1}$ and $f_{2}$, making the identification of the relevant resonance frequency $f_{\mathrm{r}}$ at least difficult. Therefore, the course of the resonance frequency $f_{\mathrm{r}}$ is no longer plotted. Instead, the quantity $L_{Y}$ from (56) is shown in Figure 16 over the frequency $f$ of the excitation and over the radius $R$ of the system.

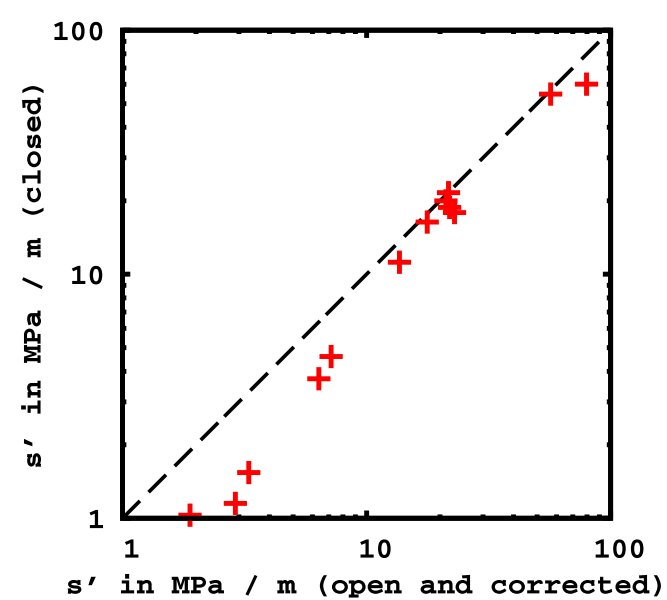

Figure 14. Dynamic stiffness $s^{\prime}$ measured with closed boundary, against dynamic stiffness measured with open boundary plus correction; line of equality (dashed).

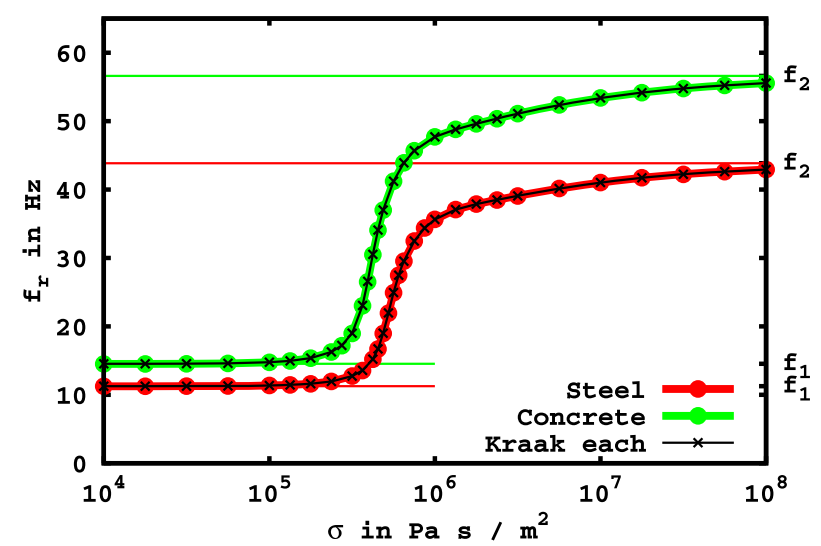

Figure 15. Shift of the resonance frequency $f_{\mathrm{r}}$ depending on the flow resistivity $\sigma$ for steel and concrete plates, lines for asymptotes at $f_{1}$ and $f_{2}$.

The perspective has been especially chosen to make the shift of the maxima in the $f-R$-domain clearly visible. A ridge at $f=f_{2}$ and $R \geq 1 \mathrm{~m}$ blocks the shift of the eigenfrequencies. Only the lowest eigenfrequencies can slightly penetrate the ridge. The yellow peak at the right occurs at $f_{1}$ which is the resonance frequency $f_{r}$ of this system for the standardized setup with $R=100 \mathrm{~mm}$.

Figures 17-22 show $L_{Y}$ - this time as top view and for different values of the flow resistivity $\sigma$ each. Figure 19 corresponds to Figure 16.

For low values of the flow resistivity $\sigma$ (Figs. 17 and 18), the eigenfrequencies reach far into the measurement region between $f_{1}$ and $f_{2}$. This effect is gradually reduced by increasing $\sigma$.

Can the dynamic stiffness, which is measured with the standardized setup, be transferred equivalently to a system of room size? The standardized prediction of a real-sized room is based on the model of a simple oscillator with rigid body motion of the upper plate, c.f. [1]. The standardized measurement is - by means of the standard's formulae (3)-(6) or Kraak's correction (22) - transferred to resting 


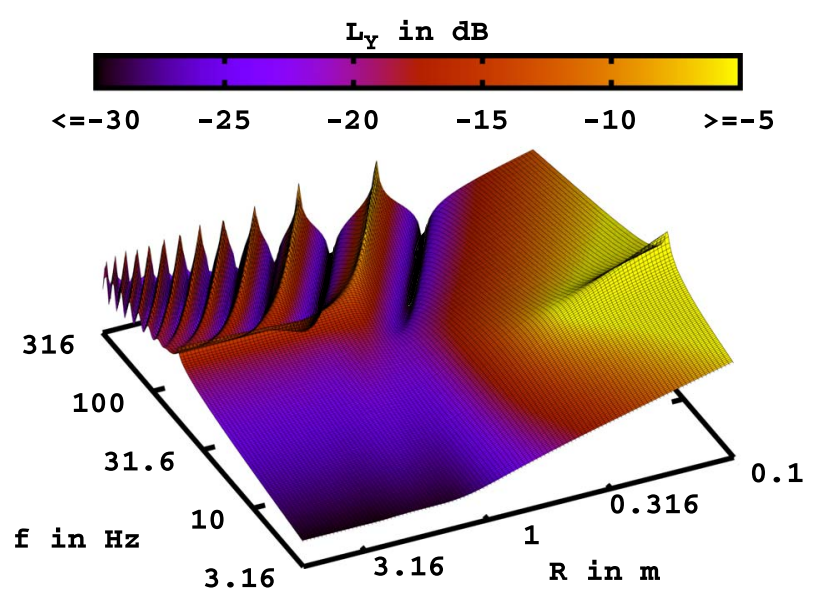

Figure 16. Level of admittance $L_{Y}$ according to (56).

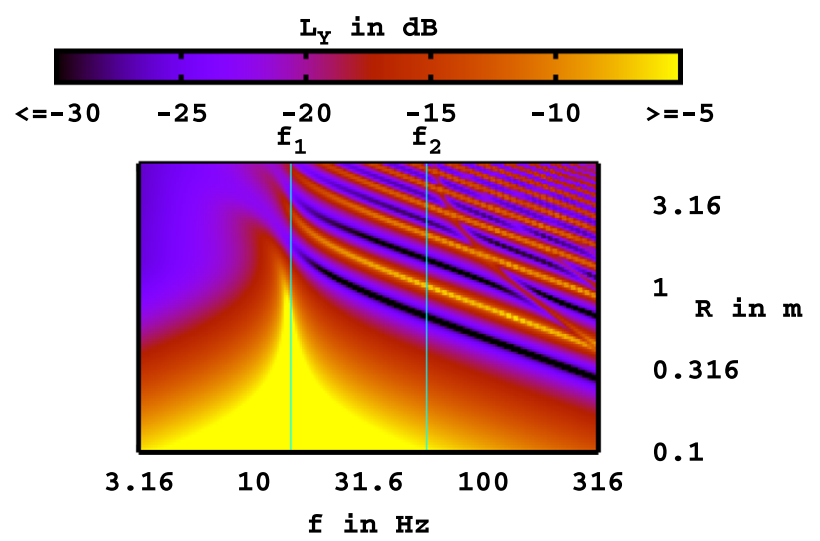

Figure 17. Level of admittance $L_{Y}$ according to (56); cyan marker lines for $f_{1}$ and $f_{2} ; \sigma=1.0 \times 10^{2} \mathrm{~Pa} \mathrm{~s} / \mathrm{m}^{2}$.

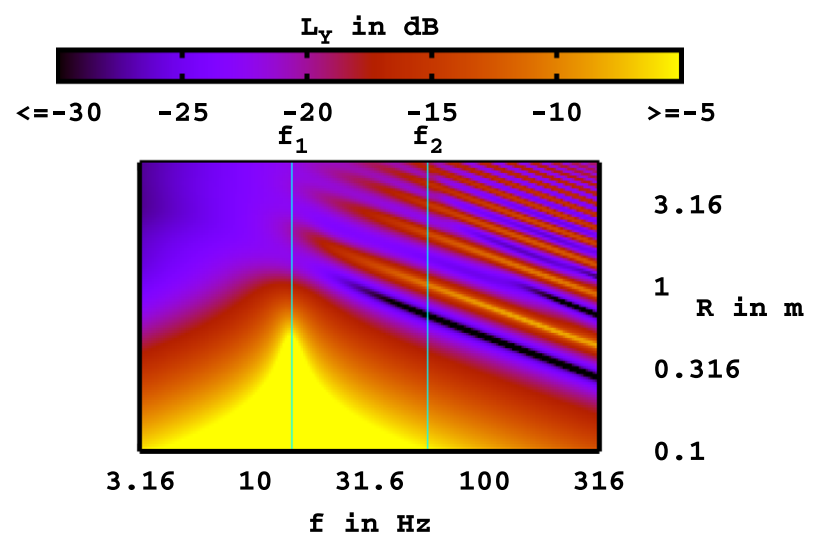

Figure 18. Level of admittance $L_{Y}$ according to (56); cyan marker lines for $f_{1}$ and $f_{2} ; \sigma=1.0 \times 10^{3} \mathrm{~Pa} \mathrm{~s} / \mathrm{m}^{2}$.

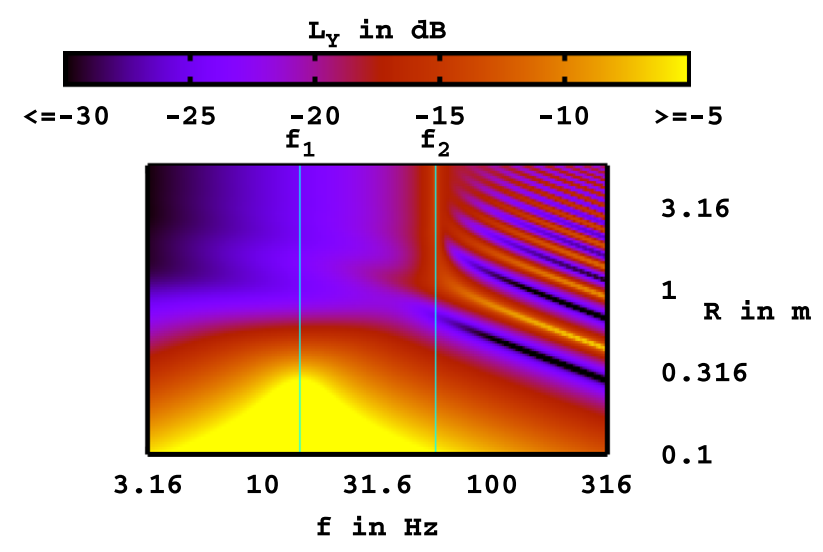

Figure 19. Level of admittance $L_{Y}$ according to (56); cyan marker lines for $f_{1}$ and $f_{2} ; \sigma=1.0 \times 10^{4} \mathrm{~Pa} \mathrm{~s} / \mathrm{m}^{2}$.

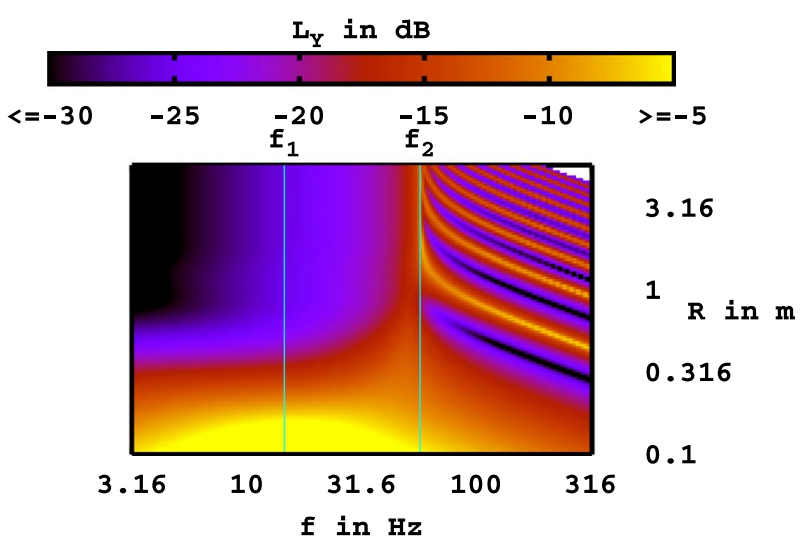

Figure 20. Level of admittance $L_{Y}$ according to (56); cyan marker lines for $f_{1}$ and $f_{2} ; \sigma=1.0 \times 10^{5} \mathrm{~Pa} \mathrm{~s} / \mathrm{m}^{2}$; small blank upper right corner due to numerical instability.

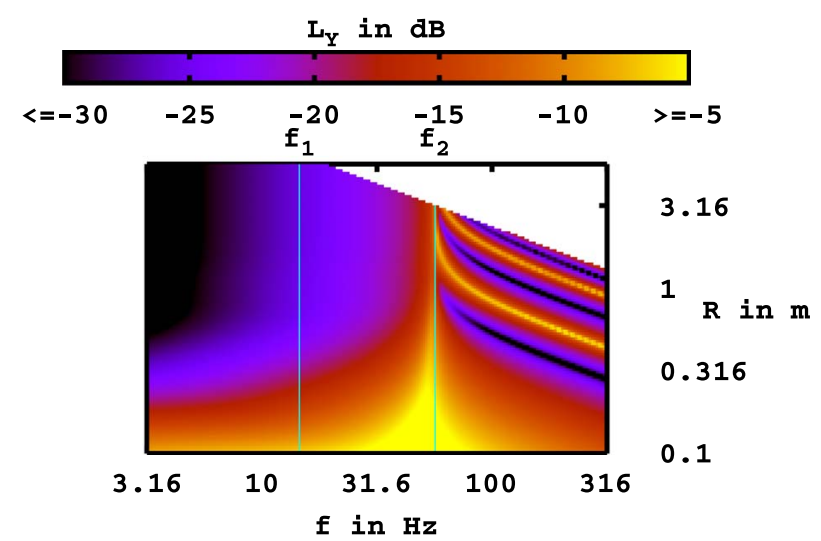

Figure 21. Level of admittance $L_{Y}$ according to (56); cyan marker lines for $f_{1}$ and $f_{2} ; \sigma=1.0 \times 10^{6} \mathrm{~Pa} \mathrm{~s} / \mathrm{m}^{2}$; large blank upper right corner due to numerical instability. 


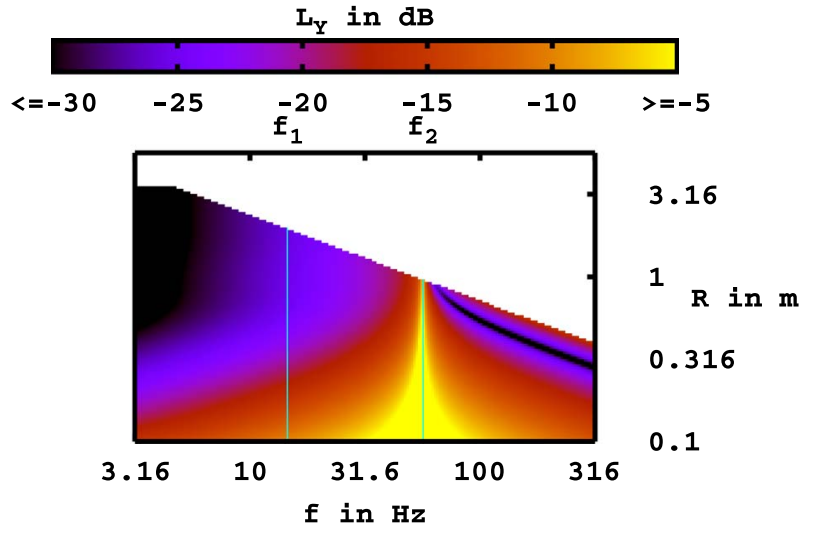

Figure 22. Level of admittance $L_{Y}$ according to (56); cyan marker lines for $f_{1}$ and $f_{2} ; \sigma=1.0 \times 10^{7} \mathrm{~Pa} \mathrm{~s} / \mathrm{m}^{2}$; large blank upper right corner due to numerical instability.

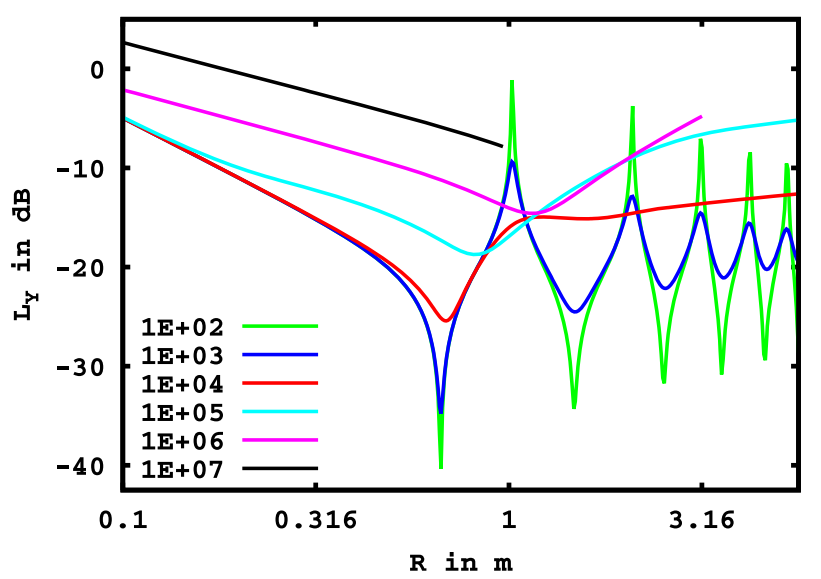

Figure 23. Level of admittance $L_{Y}$ according to (56); at frequency $f=f_{2} ; \sigma$ according to legend (unit: $\mathrm{Pa} \mathrm{s} / \mathrm{m}^{2}$ ).

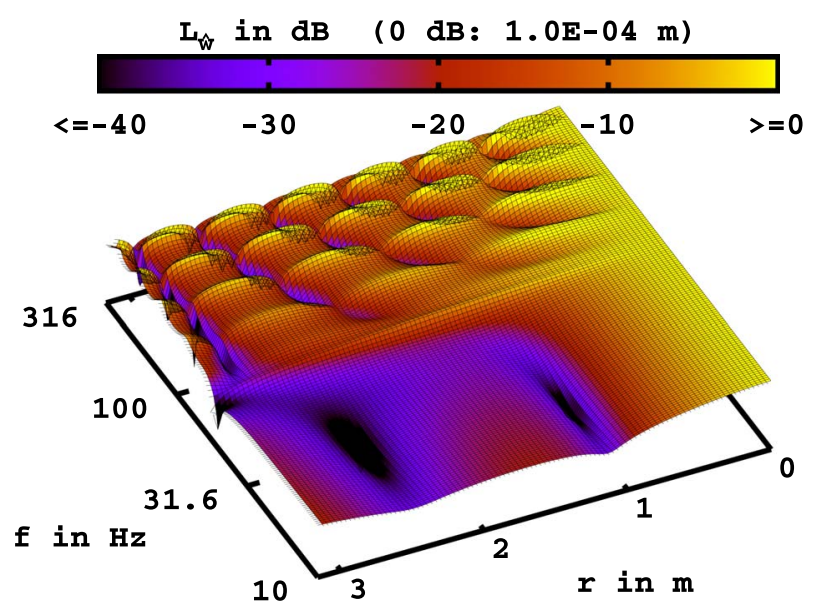

Figure 24. Deflection level $L_{\hat{w}}$, concrete plate, $R=3.16 \mathrm{~m}$, $\hat{w}_{0}=1 \times 10^{-4} \mathrm{~m}$, further values from Table 1 , open boundary according to (50). air inside the underlay and thereby to the same simple oscillator model. The relevant resonance frequency is at $f_{\mathrm{r}}=f_{2}$ in both cases.

As can be seen in Figures 17 and 18 along the cyan $f_{2}$-marker lines, different room sizes $R$ lead to heavily changing admittances at frequency $f_{2}$. For higher flow resistivity $\sigma$ (Figs. 19-22), the admittance of room sized systems at frequency $f_{2}$ is more constant, but still not the same as in the standardized setup at $R=0.1 \mathrm{~m}$. This behaviour at $f_{2}$ for different flow resistivities $\sigma$ is summarized in Figure 23 . From the excitation point of view (the input-side of the system), systems of the same materials but of different lateral dimensions behave differently in terms of admittance.

Figure 24 shows the level $L_{\hat{w}}$ of the absolute value of the deflection $|\hat{w}|$ over the spatial coordinate $r$ and the frequency $f$ for a radius $R=3.16 \mathrm{~m}$ of the system. The deflection occurs mainly in the vicinity of the excitation at $r=r_{\text {in }}$. Above frequency $f_{2}$, a modal structure along the complete $r$-coordinate is clearly visible. The case with closed outer boundary was examined, too. The only significant difference is a constant ridge along the $r$-coordinate at frequency $f_{2}$, as calculated in (54). The bending of the upper plate in real room situations is thereby obvious. This indicates that a frequency significantly above $f_{2}$ might not necessarily be sufficient for the intended vibration decoupling. Further research is needed at that point, employing a model that includes the plate of the bare floor and the sound emission into the room below (the output-side of the system).

\section{Conclusion}

Kraak's calculation was extended by the inertia of the air and a flexible upper plate. Also, adiabatic changes were preferred against isothermal changes. For the standardized setup, both calculations yield mostly identical results. The standardized evaluation procedure for the dynamic stiffness was applied to calculated results. It was shown, that the procedures of the standard and of its German preamble have significant drawbacks. In contrast, Kraak's continuous correction formula provides an easy way to achieve results very close to the exact values. The authors recommend to include Kraak's correction in a revised version of the standard, either analytically or in tabular form with significantly fine discretization.

Another option would be to standardize closed boundaries so that the air cannot leave the system. In this case, a simple formula directly yields the dynamic stiffness. But measurements indicate that fulfilling the closed boundary conditions is difficult to accomplish.

Calculations with room sized systems indicate that the dynamic stiffness cannot be transferred from the standardized measurement setup to real sized systems with flexible plates. This needs further examination.

\section{Conflict of interest}

The authors declared no conflict of interests. 


\section{Acknowledgments}

The authors would like to express their gratitude to Dr. Ernst Sonntag for providing the information that the background for the standardized airflow resistivity correction was based on the paper by Kraak [5]. The measurements of Section 6 have been conducted as part of a research project, concerning insulation materials made from renewables, which was funded by the Federal Ministry of Food and Agriculture due to a decision of the German Bundestag.

\section{References}

1. ISO 12354-2: Building acoustics - Estimation of acoustic performance of buildings from the performance of elements Part 2: Impact sound insulation between rooms (2017).

2. ISO 9052-1: Acoustics - Determination of dynamic stiffness Part 1: Materials used under floating floors in dwellings (1989).

3. DIN EN 29052-1: Akustik - Bestimmung der dynamischen Steifigkeit - Teil 1: Materialien, die unter schwimmenden Estrichen in Wohngebäuden verwendet werden (1992).
4. ISO 9053: Acoustics - Materials for acoustical applications Determination of airflow resistance (1991).

5. W. Kraak: Der Einfluss des Strömungsstandwertes bei der Bestimmung der dynamischen Steifigkeit poröser DämmMaterialien. Hochfrequenztechnik und Elektroakustik 67, 4/ 5 (1959) 111-113.

6. A. Schiavi, C. Guglielmone, P. Miglietta: Effect and importance of static-load on airflow resistivity determination and its consequences on dynamic stiffness. Applied Acoustics 72 (2011) 705-710.

7. C. Crispin, C. Mertens, J. Medved: Measurement of the dynamic stiffness of porous materials taking into account their airflow resistivity. Building Acoustics 21, 3 (2014) 221233.

8. P. Guidorzi, L. Barbaresi, M. Garai: Measuring the dynamic stiffness of resilient materials using ESS and MLS signals. Applied Acoustics 138 (2018) 92-100.

9. DEGA-Memorandum: Tieffrequente Schallübertragung von schwimmenden Estrichen, 2020, https://www.dega-akustik. de/fileadmin/dega-akustik.de/publikationen/dega_br_0106. pdf, last visited 12. Jan. 2021.

10. S. Timoshenko, S. Woinowsky-Krieger: Theory of Plates and Shells, 2nd ed. McGraw-Hill, New York, 1959.

11. A. Nádai: Die elastischen Platten. Springer, Berlin, 1968.

12. GNU Multiple Precision Arithmetic Library. https://gmplib. org/ and ftp.gnu.org/gnu/gmp, both last visited 02. Nov. 2020.

Cite this article as: Schmelzer M, Wittstock V, Bietz H \& Brezas S. 2021. On the influence of the air flow resistivity on the measurement of the dynamic stiffness of underlays for floating floors. Acta Acustica, 5, 13. 